\title{
Pressure dependence of the Griffiths-like phase in 5:4 intermetallics
}

\author{
N. Marcano $\odot,{ }^{1,2,{ }^{*}}$ P. A. Algarabel, ${ }^{2,3}$ J. Rodríguez Fernández $\odot,{ }^{4}$ J. P. Araujo $\odot,{ }^{5}$ A. M. Pereira, ${ }^{5}$ \\ J. H. Belo, ${ }^{5}$ C. Magén, ${ }^{2,3}$ L. Morellón $\odot,{ }^{2,3}$ and M. R. Ibarra ${ }^{2,3}$ \\ ${ }^{1}$ Centro Universitario de la Defensa, Academia General Militar Crta. Huesca s/n, 50090 Zaragoza, Spain \\ ${ }^{2}$ Instituto de Nanociencia y Materiales de Aragón, CSIC-Universidad de Zaragoza, 50009 Zaragoza, Spain \\ ${ }^{3}$ Dpto. Física de la Materia Condensada, Universidad de Zaragoza, 50009 Zaragoza, Spain \\ ${ }^{4}$ Dpto. CITIMAC, Universidad de Cantabria, 39005 Santander, Spain \\ ${ }^{5}$ IFIMUP Institute of Physics for Advanced Materials, Nanotechnology and Photonics, Physics and Astronomy Department, \\ University of Porto, Rua do Campo Alegre s/n, 4169-007 Porto, Portugal
}

(Received 1 July 2020; accepted 19 October 2020; published 9 November 2020)

\begin{abstract}
We report a study of the effect of hydrostatic pressure $(P)$ on the Griffiths-like phase in selected compounds of the $R_{5}\left(\mathrm{Si}_{x} \mathrm{Ge}_{1}-x\right)_{4}$ family of alloys $\left(\mathrm{Tb}_{4.925} \mathrm{La}_{0.075} \mathrm{Si}_{2} \mathrm{Ge}_{2}\right.$ and $\left.\mathrm{Gd}_{5} \mathrm{Ge}_{4}\right)$ which present either the $\mathrm{Gd}_{5} \mathrm{Si}_{2} \mathrm{Ge}_{2}-$ type (monoclinic, M) or the $\mathrm{Sm}_{5} \mathrm{Ge}_{4}$-type [orthorhombic-II, $O$ (II)] structural phases at room temperature. The downward deviation in the inverse low-field dc susceptibility $\chi_{d c}^{-1}$ from the Curie-Weiss law below a characteristic temperature $T_{G}$ indicates that the Griffiths-like phase exists at pressures up to 10 kbar. From the obtained $T-P$ phase diagrams, the pressure coefficient of the Griffiths-like temperature, $d T_{G} / d P$, has been determined. These results are compared with those obtained in $\mathrm{Dy}_{5} \mathrm{Si}_{3} \mathrm{Ge}$ in a previous work. The $d T_{G} / d P$ coefficient is strongly dependent on the nature (first or second order) of the long-range order (FM or AFM) transition. This effect can be ascribed to a different structural character of the clusters within the Griffiths phase. A ratio of $\sim 0.5$ between the $d T_{G} / d P$ and the pressure coefficient of long-range magnetic ordering temperatures, $d T_{C, N} / d P\left(T_{C}\right.$, ferromagnetic; $T_{N}$, antiferromagnetic), is found in all the studied compounds.
\end{abstract}

DOI: 10.1103/PhysRevB.102.174416

\section{INTRODUCTION}

The Griffiths phase (GP) is a particular magnetic state characterized by the formation of short-range magnetically ordered regions (clusters) within the paramagnetic phase (PM) at some well-defined temperature $T_{G}$, well above that of long-range magnetic ordering $\left(T_{\text {order }}\right)$. This phase, stable in the temperature region $T_{\text {order }}<T<T_{G}$, first postulated by Griffiths in the context of random site dilution in Ising ferromagnets [1] and later extended to magnetic systems containing any bond distribution [2], is characterized by the nonanalytical behavior of thermodynamic properties (e.g., magnetization). Here, $T_{G}$ refers to the ordering temperature of the nondiluted system (or pure ferromagnetic phase) and is the temperature at which the GP forms (Griffiths temperature), whereas $T_{\text {order }}$ is the long-range ordering temperature of the randomly diluted ferromagnet.

To date, the existence of GP has been shown in materials as diverse as transition metal oxides [3,4], magnetic semiconductors [5,6], metallic quantum ferromagnets [7], and rare earth intermetallic compounds [8] including magnetocaloric intermetallics [9-12], among others. In particular, the presence of GP in several compounds of the $R_{5}\left(\mathrm{Si}_{x} \mathrm{Ge}_{1-x}\right)_{4}$ family of magnetocaloric materials has attracted much attention $[9,12,13]$. The appearance of GP arises from the local disorder within the crystallographic structures and the

*marcanon@unizar.es strong interplay between structure and magnetism present in these materials. The remarkable properties of this family of compounds are associated with its intrinsically layered crystal structure, which is built by stacking two-dimensional sub-nanometer-thick layers via partially covalent interslab $\mathrm{Si}(\mathrm{Ge})-\mathrm{Si}(\mathrm{Ge})$ bonds [14]. The crystallographic phase and the nature of the magnetic interactions are controlled by a number of interslab covalentlike $\mathrm{Si}(\mathrm{Ge})-\mathrm{Si}(\mathrm{Ge})$ bonds. Three different crystal structures are observed at room temperature depending on the $\mathrm{Si} / \mathrm{Ge}$ ratio, which are intimately related to the number of formed interslab covalentlike $\mathrm{Si}(\mathrm{Ge})-\mathrm{Si}(\mathrm{Ge})$ bonds (see Fig. 1): the $\mathrm{Gd}_{5} \mathrm{Si}_{4}$-type orthorhombic structure [also referred to as the $O(\mathrm{I})$ structure with space group Pnma] where all $\mathrm{Si}(\mathrm{Ge})-\mathrm{Si}(\mathrm{Ge})$ bonds are formed, the $\mathrm{Sm}_{5} \mathrm{Ge}_{4}$-type structure [referred to as the orthorhombic-II, $O$ (II) structure with space group Pnma] with no interslab $\mathrm{Si}(\mathrm{Ge})-\mathrm{Si}(\mathrm{Ge})$ bonds, and the $\mathrm{Gd}_{5} \mathrm{Si}_{2} \mathrm{Ge}_{2}$-type monoclinic crystal structure (M state, space group $P 112_{1} / a$ ) where every other $\mathrm{Si}(\mathrm{Ge})-\mathrm{Si}(\mathrm{Ge})$ bond is broken.

By means of a thorough study of the magnetic properties of the $R_{5}\left(\mathrm{Si}_{x} \mathrm{Ge}_{1-x}\right)_{4}$ system, with $R=\mathrm{Gd}, \mathrm{Tb}$, Dy, and $\mathrm{Ho}$, Pereira and co-workers reconstructed the $\mathrm{Si} / \mathrm{Ge}$ doping-temperature $(x, T)$ magnetic and crystallographic phase diagrams of these family of compounds [12]. They found that a Griffiths-like behavior always appeared in the compositions which present the $\mathrm{M}$ or the $O$ (II) structural phases at room temperature. According to their results, $T_{G}$ was identified as the Curie temperature of the Si-rich compound region [which in this case has an orthorhombic $O(\mathrm{I})$ structure]. 


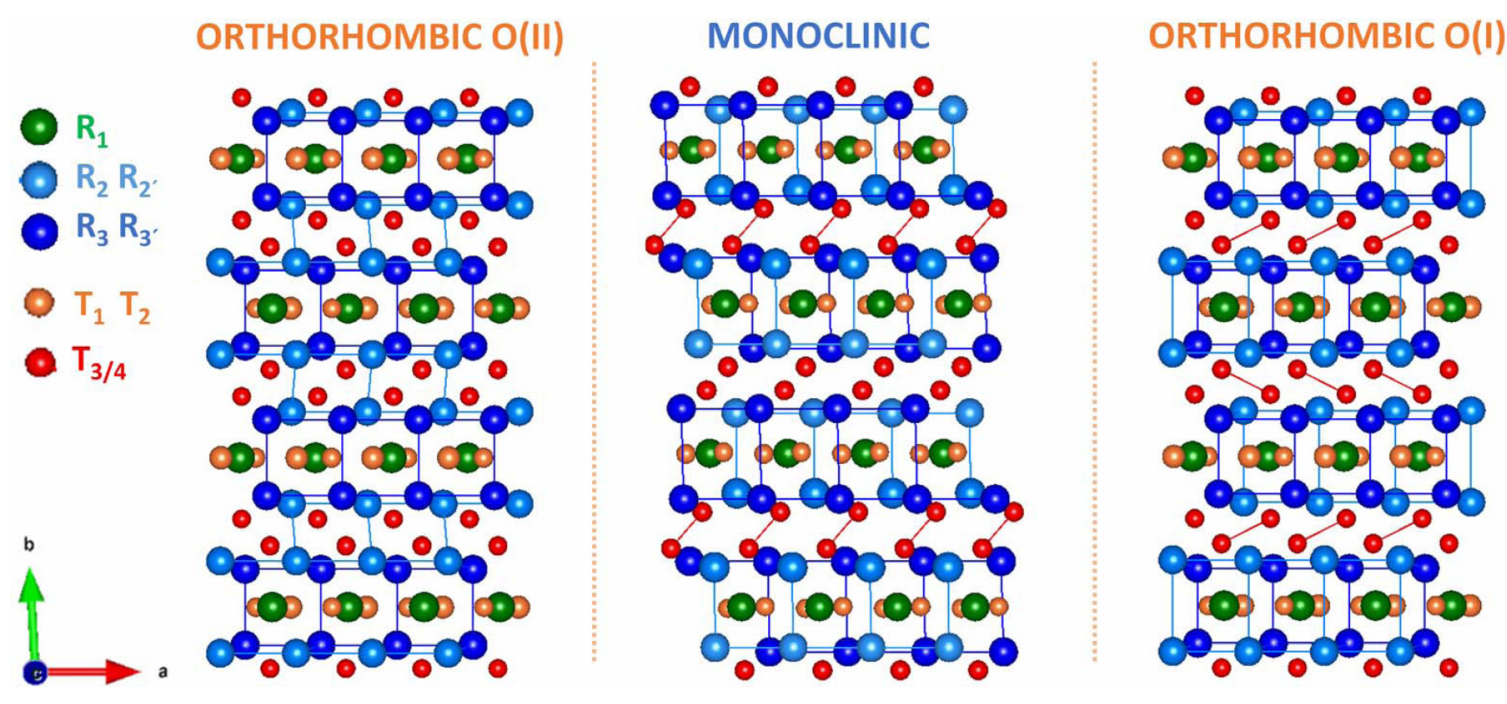

FIG. 1. Three structure types found at room temperature in $R_{5}\left(\mathrm{Si}_{x} \mathrm{Ge}_{1-x}\right)_{4}$ : (left) $\mathrm{Sm}_{5} \mathrm{Ge}_{4}$-type $[O(\mathrm{II})]$; (middle) $\mathrm{Gd}_{5} \mathrm{Si}_{2} \mathrm{Ge}{ }_{2}$-type $(\mathrm{M})$; (right) $\mathrm{Gd}_{5} \mathrm{Si}_{4}$-type $[O(\mathrm{I})]$. The $R$ atoms occupying different sites are shown using green $\left(R_{1}\right)$, light blue $\left(R_{2}\right)$, and dark blue $\left(R_{3}\right)$ spheres. The orange circles represent the $\mathrm{Si} / \mathrm{Ge}$ atoms located at the $T_{1}, T_{2}$ positions within the slab. The red circles represent the $\mathrm{Si} / \mathrm{Ge}$ atoms responsible for bonding between slabs, located at the $T_{3}, T_{4}$ positions. The thick red lines indicate the $\mathrm{Si}(\mathrm{Ge})-\mathrm{Si}(\mathrm{Ge})$ covalently bonded pairs of atoms.

That observation suggested that, when the system is in the $\mathrm{M}$ or $O(\mathrm{II})$ phases, it retains memory of the $O(\mathrm{I})$ phase (i.e., pure state) signaled by an anomaly at $T_{G}$. This scenario was previously proposed by Magen et al. for the $\mathrm{Tb}_{5} \mathrm{Si}_{2} \mathrm{Ge}_{2}$ [9]. In addition, they found a universal scaling of the $\mathrm{Si} / \mathrm{Ge}$ doping ( $x-T)$ phase diagrams for the $R_{5}\left(\mathrm{Si}_{x} \mathrm{Ge}_{1-x}\right)_{4}$ systems. Those studies indicated that the rupture of the interslab $\mathrm{Si}(\mathrm{Ge})$ $\mathrm{Si}(\mathrm{Ge})$ bonds is the key feature for the appearance of the GP in these systems and, thus, a percolative mechanism was behind the observed universality [12].

Apart from tuning the $\mathrm{Si} / \mathrm{Ge}$ doping (chemical pressure), the distance and chemical bonding between the neighboring slabs can also be modified by changing the $R$ ions (also chemical pressure) or by changing external parameters such as temperature $T$, magnetic field $H$, or hydrostatic pressure $P$. In particular, the extraordinary sensitivity of the electronic and crystal structures to the application of hydrostatic pressure have led to a systematic investigation $[15,16]$ in order to thoroughly comprehend the microscopic processes taking place in the 5:4 materials. To date, hydrostatic pressure effects on the true long-range ordered state and first-order structural transitions have been reported. However, less research has been devoted to the effects of pressure on the short-range ordered state (GP) [17].

Here, we investigate the effect of hydrostatic pressure on the short-range magnetic correlations in the $\mathrm{M}$ and $O(\mathrm{II})$ structures for the 5:4 systems. For this purpose we have studied the temperature-pressure $(T-P)$ magnetic phase diagrams of two selected 5:4 compounds: (i) $\mathrm{Tb}_{4.925} \mathrm{La}_{0.075} \mathrm{Si}_{2} \mathrm{Ge}_{2}$ as representative of a monoclinic Griffiths phase (M-GP) with a first order magnetic-structural M-PM $\rightarrow O(\mathrm{I})$-FM transition [18-20] and (ii) $\mathrm{Gd}_{5} \mathrm{Ge}_{4}$ as an example of a orthorhombic $O$ (II) Griffiths phase [O(II)-GP] with a second order $O$ (II)-PM $\rightarrow O$ (II)-AFM transition [21]. To address this, we have performed a complete study of the magnetic properties by measuring low-field dc magnetization as a function of temperature and hydrostatic pressure. We compare our results with those obtained in $\mathrm{Dy}_{5} \mathrm{Si}_{3} \mathrm{Ge}$ in a previous work [17], as a representative 5:4 compound of M-Griffiths with a second order M-PM $\rightarrow$ M-AFM transition. These three 5:4 compounds were chosen because they cover the main types of long-range magnetic and structural transitions observed in this family of compounds. Our experiments indicate that the effect of pressure on the Griffiths-like transition temperature strongly depends on the nature (first or second order) of the main long-range order transition in the system. Such a different response is proposed to be due to different structural character of the clusters within the Griffiths phase.

\section{EXPERIMENTAL DETAILS}

The polycrystalline samples studied here were prepared using an arc-melting furnace. The starting elements were 99.99 wt. \% pure Tb, 99.9 wt. \% pure Gd, and 99.9999 wt. \% pure Si and Ge (Alfa Aesar). The quality of the as-cast samples was checked by room-temperature $\mathrm{x}$-ray diffraction and scanning electron microscopy. Further details on sample preparation and basic structural characterization at room temperature can be found elsewhere [20,22,23].

Magnetic measurements were performed in a commercial (Quantum Design) superconducting quantum interference device (SQUID) magnetometer in applied dc magnetic fields from $H_{d c}=20$ Oe up to $H_{d c}=1 \mathrm{kOe}$ in the temperature range $2-300 \mathrm{~K}$. For zero-field-cooled (ZFC) measurements of the magnetization, the sample was cooled from the PM state in a zero applied field and the magnetization was measured while warming the sample. The magnetization data in the field-cooled state were collected while cooling the sample (FCC) in an applied field. Data were also collected while warming the sample in the presence of field after cooling in the same field (FCW).

Pressure-dependent experiments were carried out using a commercial miniature piston-cylinder-type $\mathrm{CuBe}$ Mcell 10 by EasyLab [24]. The pressure value was determined at low 

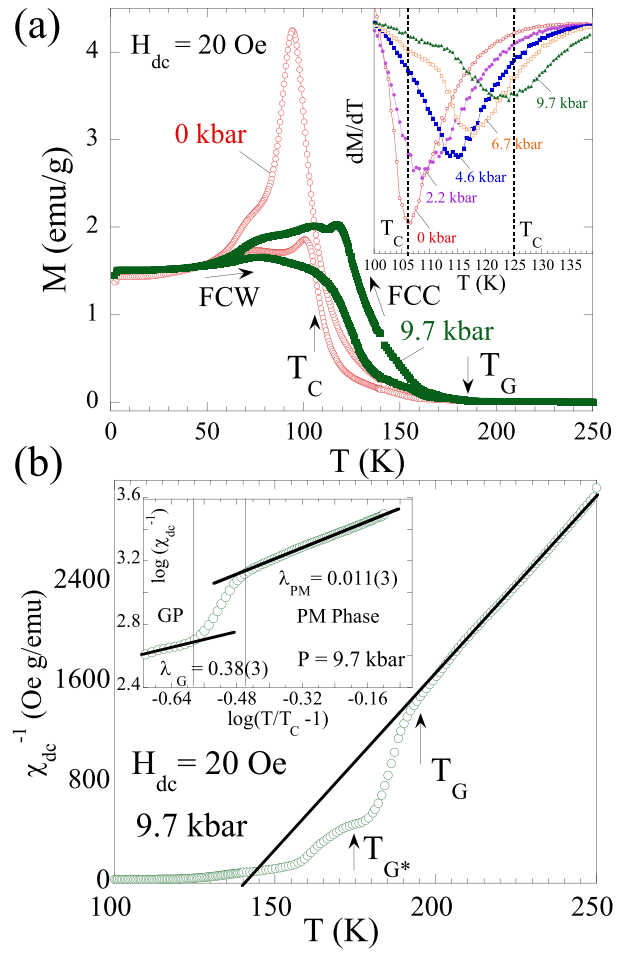

(d)

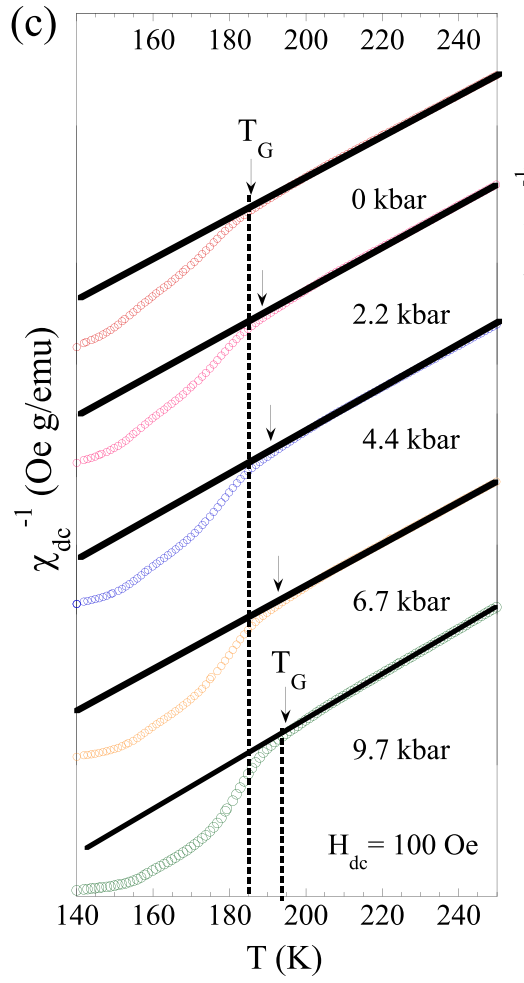

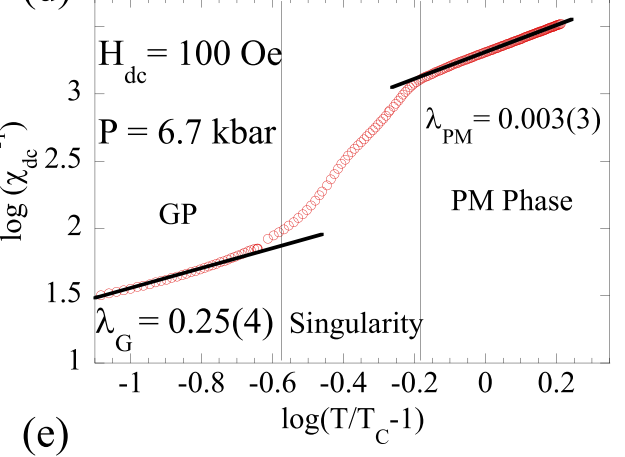

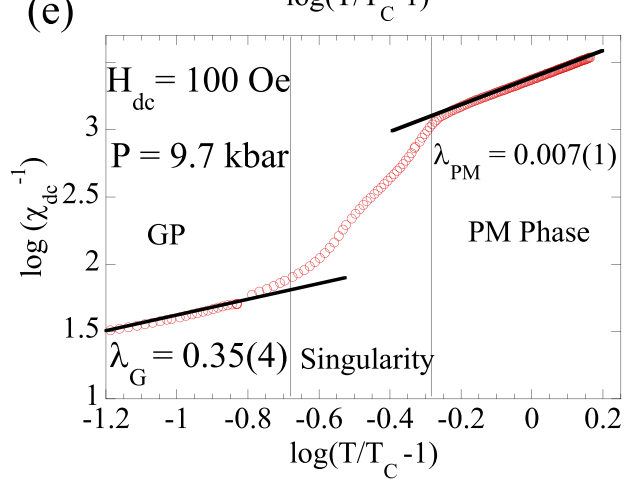

FIG. 2. Magnetization measurements as a function of temperature and hydrostatic pressure in $\mathrm{Tb}_{4.925} \mathrm{La}_{0.075} \mathrm{Si}_{2} \mathrm{Ge}_{2}$. (a) FCC and $\mathrm{FCW}$ magnetizations as a function of temperature measured in an applied field of 20 Oe at $P=0$ kbar (open circles) and under pressure of $9.7 \mathrm{kbar}$ (filled squares). Inset: $d M / d T$ at selected hydrostatic pressures. For clarity only the values upon heating are displayed. (b) Temperature dependence of $\chi_{d c}^{-1}(T)$ at 20 Oe at 9.7 kbar. The inset shows the linear fit of $\chi_{d c}^{-1}$ vs $\left(T / T_{C}-1\right)$ in double logarithmic scale in the Griffiths-like and PM phases, respectively. (c) $\chi_{d c}^{-1}(T)$ at $100 \mathrm{Oe}$ at selected hydrostatic pressures. The curves have been vertically displaced for clarity. Thick solid lines depict the fit to the Curie-Weiss law at the PM state, and the arrows show deviation from the CW law below the Griffiths temperature $T_{G}$ in each case. (d),(e) Linear fit of $\chi_{d c}^{-1}$ vs $\left(T / T_{C}-1\right)$ in double logarithmic scale at 100 Oe near $T_{G}$ at selected hydrostatic pressures (6.7 and $9.7 \mathrm{kbar}$, respectively).

temperature using the known pressure dependence of the critical temperature of the superconducting state of a tin (Sn) sensor placed within the cell. The sample and the Sn manometer were compressed in a Teflon capsule filled with a liquid pressure-transmitting medium (a mixture of mineral oils). Technical details about the pressure cell can be found in Ref. [24]. One should note that the medium and the cell present a much smaller diamagnetic signal when compared to the sample, and that such a signal is constant with temperature, thus not interfering with the main conclusions of the present work. The magnetization was measured under hydrostatic pressures up to $10 \mathrm{kbar}$.

\section{RESULTS AND DISCUSSION}

Figure 2(a) displays the FCC and FCW magnetization measurements of $\mathrm{Tb}_{4.925} \mathrm{La}_{0.075} \mathrm{Si}_{2} \mathrm{Ge}_{2}$ as a function of hydrostatic pressure at low field $\left(H_{d c}=20 \mathrm{Oe}\right)$. Only ambient and maximum pressures are depicted for the sake of clarity. At ambient pressure, the ferromagnetic transition $\mathrm{M}-\mathrm{PM} \rightarrow O(\mathrm{I})$-FM (obtained by the minimum of the numerical derivative, $d M / d T)$ occurs at $T_{C}=100(1) \mathrm{K}$ in the FCC magnetization, whereas $T_{C}=106(1) \mathrm{K}$ in the FCW magnetization. The thermal hysteresis between both FCC and FCW data in the vicinity of $T_{C}$ signals a first-order transformation in this temperature range. Previous studies on the parent compound $\mathrm{Tb}_{5} \mathrm{Si}_{2} \mathrm{Ge}_{2}$ [19] reported a first-order structural transition $O(\mathrm{I}) \rightarrow \mathrm{M}$ approximately $10 \mathrm{~K}$ below $T_{C}$. The observed hysteresis around $T_{C}$ indicates that the magnetic and structural transitions $O(\mathrm{I}) \rightarrow \mathrm{M}$ and FM $\rightarrow$ PM occur simultaneously in this La-diluted compound. Such a hysteresis is observed at the highest pressure as well, which indicates that the transition retains its first-order character within the pressure range studied. The derivative of the magnetization $d M / d T$ measured upon warming for selected pressures is depicted as an inset in order to show the effect of the applied pressure on the position of the minimum (i.e., the $T_{C}$ ). The minimum shifts towards higher temperatures from $T_{C}=106(1) \mathrm{K}$ at ambient pressure to $T_{C}=124(1) \mathrm{K}$ at the highest applied pressure (see Table I).

The anomaly observed at $T_{S R}=65 \mathrm{~K}$ on the $M(T)$ curve at ambient pressure is assigned to a spin reorientation process similar to that observed in the parent compound $\mathrm{Tb}_{5} \mathrm{Si}_{2} \mathrm{Ge}_{2}$ [25,26]. An increase of $T_{S R}$ with the applied pressure is observed reaching a value of $70 \mathrm{~K}$ at the highest measured pressure (see Table I).

The onset of a Griffiths-like phase at a characteristic temperature $T_{G}=185(1) \mathrm{K}$ has been reported previously in this compound by means of the anomalous behavior on the temperature dependence of the inverse of the magnetic susceptibility $\chi_{d c}^{-1}(T)$ at low fields $\left(<500\right.$ Oe) [27]. Here, $T_{G}$ is defined as the temperature where the deviation from the 
TABLE I. Magnetic properties of the $\mathrm{Tb}_{4.925} \mathrm{La}_{0.075} \mathrm{Si}_{2} \mathrm{Ge}_{2}$ system obtained from the analysis of the low-field dc magnetic susceptibility at selected hydrostatic pressure values: the Curie temperature $T_{C}$, the spin reorientation transition $T_{S R}$, the Griffiths temperature $T_{G}$, the paramagnetic Curie temperature $\theta_{P}$, the susceptibility exponent $\lambda_{G}$, and the effective magnetic moment per $\mathrm{Tb}^{3+}$ ion $\mu_{\mathrm{eff}}^{P M} / \mathrm{Tb}^{3+}\left(\mu_{B}\right)$.

\begin{tabular}{lcccccc}
\hline \hline $\begin{array}{l}P \\
\text { (kbar) }\end{array}$ & $\begin{array}{c}T_{S R} \\
(\mathrm{~K})\end{array}$ & $\begin{array}{c}T_{C} \\
(\mathrm{~K})\end{array}$ & $\begin{array}{c}T_{G} \\
(\mathrm{~K})\end{array}$ & $\begin{array}{c}\theta_{P} \\
(\mathrm{~K})\end{array}$ & $\lambda_{G}$ & $\begin{array}{c}\mu_{\mathrm{eff}}^{P M} / \mathrm{Tb}^{3+} \\
\left(\mu_{B}\right)\end{array}$ \\
\hline 0 & 65 & 106 & 185 & 106 & $0.22(1)$ & $9.7(1)$ \\
2.2 & 66 & 110 & 187 & 110 & $0.16(1)$ & $9.5(1)$ \\
4.4 & 67 & 115 & 190 & 113 & $0.20(1)$ & $9.5(1)$ \\
6.7 & 68 & 118 & 192 & 114 & $0.25(4)$ & $9.5(1)$ \\
9.7 & 70 & 124 & 195 & 121 & $0.35(4)$ & $9.2(1)$ \\
\hline \hline
\end{tabular}

typical Curie-Weiss linear behavior starts, following the criteria established in the seminal work by Magen et al. [9].

At very low fields $(<100 \mathrm{Oe})$ an additional stairlike fall in $\chi_{d c}^{-1}(T)$ was reported below a characteristic temperature $T_{G^{*}} \sim 156(1) \mathrm{K}$. This anomaly defined two different plateaus in the $\chi_{d c}^{-1}(T)$ in the PM regime which were associated to different stages of the FM clustering of spins within the GP [27]. The Griffiths singularity was characterized by means of the predicted temperature dependence of the susceptibility for a Griffiths-like phase in $f$-electron compounds:

$$
\chi_{d c}^{-1}(T) \propto\left(T / T_{C}-1\right)^{1-\lambda},
$$

where $T_{C}$ denotes the critical temperature and $(1-\lambda)$ is the effective exponent with $0 \leqslant \lambda<1$ [28]. The values obtained for the susceptibility exponent at ambient pressure were $\lambda_{P M} \approx 0$ for the conventional PM phase $\left(T>T_{G}\right)$ and $\lambda_{G} \sim 0.27(2)$ in the anomalous region $T_{G^{*}}<T<T_{G}$. Those fits have been published elsewhere (see Marcano et al. [27] for details).

The effect of hydrostatic pressure on the onset of the GP in the PM regime is analyzed in this work through the susceptibility exponent $\lambda_{G}$ and the GP temperature $T_{G}$. Figure 2(b) displays the $\chi_{d c}^{-1}(T)$ at 20 Oe at the highest applied pressure (9.7 kbar) as representative of this study. Both stairlike jumps in low field $\chi_{d c}^{-1}(T)$ below $T_{G}$ and $T_{G^{*}}$ are observed for all the measured pressures. We have fitted the logarithmic representation of $\chi_{d c}^{-1}(T)$ for each pressure to obtain the susceptibility exponent $\lambda_{G}$. It is noteworthy that for each analysis the shift of $T_{C}$ with pressure has been taken into account. According to Marcano et al., the fit of the low field $\chi_{d c}^{-1}(T)$ has been done in the temperature regime $T_{G^{*}}<T<T_{G}$ to avoid the contribution of the lower-temperature anomaly in $\chi_{d c}^{-1}(T)$ [27]. The inset of Fig. 2(b) shows the fit for the highest applied pressure $(9.7 \mathrm{kbar})$ as a representative of this study. The obtained values for $\lambda_{G}$ in this anomalous temperature regime $\left[\lambda_{G}=0.36(2)\right.$ for $6.7 \mathrm{kbar}$ and $\lambda_{G}=0.38(3)$ for $\left.9.7 \mathrm{kbar}\right]$ indicate that GP remains at higher pressures up to $9.7 \mathrm{kbar}$.

At higher field $\left(H_{d c}=100 \mathrm{Oe}\right)$ the anomaly at $T_{G^{*}}$ in $\chi_{d c}^{-1}(T)$ at ambient pressure is suppressed and the linear fit of the logarithmic representation of $\chi_{d c}^{-1}$ can be extended down to $\sim T_{C}\left(T_{C}<T<T_{G}\right)$ [27]. Figure 2(c) shows $\chi_{d c}^{-1}(T)$ curves at 100 Oe under selected pressures. The negative deviation from the Curie-Weiss law at $T_{G}$ is observed for all the measured pressures. The linear fits of the logarithmic representation of $\chi_{d c}^{-1}$ are shown in Figs. 2(d) and 2(e) for selected pressures $(P=6.7$ and $9.7 \mathrm{kbar}$, respectively) as representative of this study. The corresponding values of the exponent $\lambda_{G}$ obtained for all the measured pressures are summarized in Table I. A slight variation of $\lambda_{G}$ is observed in the investigated pressure range $\left[\lambda_{G}=0.22(1)\right.$ at $0 \mathrm{kbar}, \lambda_{G}=0.20(1)$ at $4.4 \mathrm{kbar}$, and $0.35(4)$ at $9.7 \mathrm{kbar}]$. This variation, however, is within the experimental error. The data for the different pressures follow the Curie-Weiss law above $T_{G}$ [see solid line in Fig. 2(c) ] with nearly equal effective magnetic moment $\mu_{\text {eff }}$, but different paramagnetic Curie temperature $\theta_{P}$ (see Table I). The value of $\theta_{P}$ increases as hydrostatic pressure increases, indicative of an enhancement of FM interactions.

With the application of hydrostatic pressure (i.e., as the cell volume decreases), the value of $T_{G}$ increases from $T_{G}=185(1) \mathrm{K}$ at ambient pressure up to $T_{G}=195(1) \mathrm{K}$ at $9.7 \mathrm{kbar}$. An increase of $T_{G}$ has been also observed in the $\mathrm{Tb}_{5}\left(\mathrm{Si}_{x} \mathrm{Ge}_{1-x}\right)_{4}$ system when reducing the cell volume by means of chemical pressure [either substituting $\mathrm{Tb}$ ion with $\mathrm{La}[20]$ or tuning $\mathrm{Si} / \mathrm{Ge}$ content $(x)[12,29]]$. It is worth noting that in those studies the negative deviation in $\chi_{d c}^{-1}(T)$ (i.e., the GP fingerprint) only appeared within a compositional window $0<x<0.5$ (i.e., only were observed within a determined cell volume range). Unlike those cases, in this work we observe that the negative deviation in $\chi_{d c}^{-1}(T)$ is enhanced as hydrostatic pressure increases [Fig. 2(c)]. According to previous work by Ouyang [13], such an enhancement can be related to a rise in either the size or amount of FM clusters in this compound. Thus our results indicate that reducing the cell volume by means of hydrostatic pressure enhances the GP in the investigated pressure range. The pressure dependence of the transition temperatures $T_{S R}, T_{C}$, and $T_{G}$ obtained from the data presented in Fig. 2 will be discussed below.

Figure 3 displays the main results obtained from the study carried out in $\mathrm{Gd}_{5} \mathrm{Ge}_{4}$ at $H_{d c}=50$ Oe. Figure 3(a) illustrates the FC (i.e., the magnetization data in the field-cooled state measured as warming) and ZFC magnetization measurements obtained in an applied field of 50 Oe at ambient and maximum pressures. The second order transition $O$ (II)-PM $\rightarrow$ $O$ (II)-AFM occurs at $T_{N}=130(1) \mathrm{K}$ at ambient pressure [see inset of Fig. 3(a)], in agreement with previous works [10,30]. The inset of Fig. 3(a) indicates that $T_{N}$ shifts towards higher temperatures up to $T_{N}=138(1) \mathrm{K}$ at the highest applied pressure $P=10.2 \mathrm{kbar}$ in agreement with previous work [23].

$\chi_{d c}^{-1}(T)$ at ambient pressure as a function of magnetic field is illustrated in Fig. 3(b). The 50 Oe data follow the CurieWeiss law above $\sim 240 \mathrm{~K}$ with $\theta_{P}=120(1) \mathrm{K}$ and $\mu_{\text {eff }}=$ 8.0(1) $\mu_{B} / \mathrm{Gd}^{3+}$, which is close to $7.94 \mu_{B}$ expected for a free $\mathrm{Gd}^{3+}$ ion. The large and positive value of $\theta_{P}$ indicates that the FM intraslab interactions are dominant compared to the AFM interslab interactions in agreement with previous studies $[10,30]$. The downward deviation in $\chi_{d c}^{-1}(T)$ at a characteristic temperature $T_{G}=237(1) \mathrm{K}$ well above $T_{N}$ indicates the onset of the GP, in agreement with previous works [12,13].

The inset of Fig. 3(b) displays the linear fit of $\chi_{d c}^{-1}$ vs $\left(T / T_{C}-1\right)$ in double logarithmic scale. Taking $\theta_{P}=$ $120 \mathrm{~K}$ as the ordering $T_{C}$, we find that $\lambda_{G}=0.21(5)$ for $T_{N}<$ $T<T_{G}$ for ambient pressure and $H_{d c}=50 \mathrm{Oe}$. The figure shows that when the field is increased the GP becomes less 

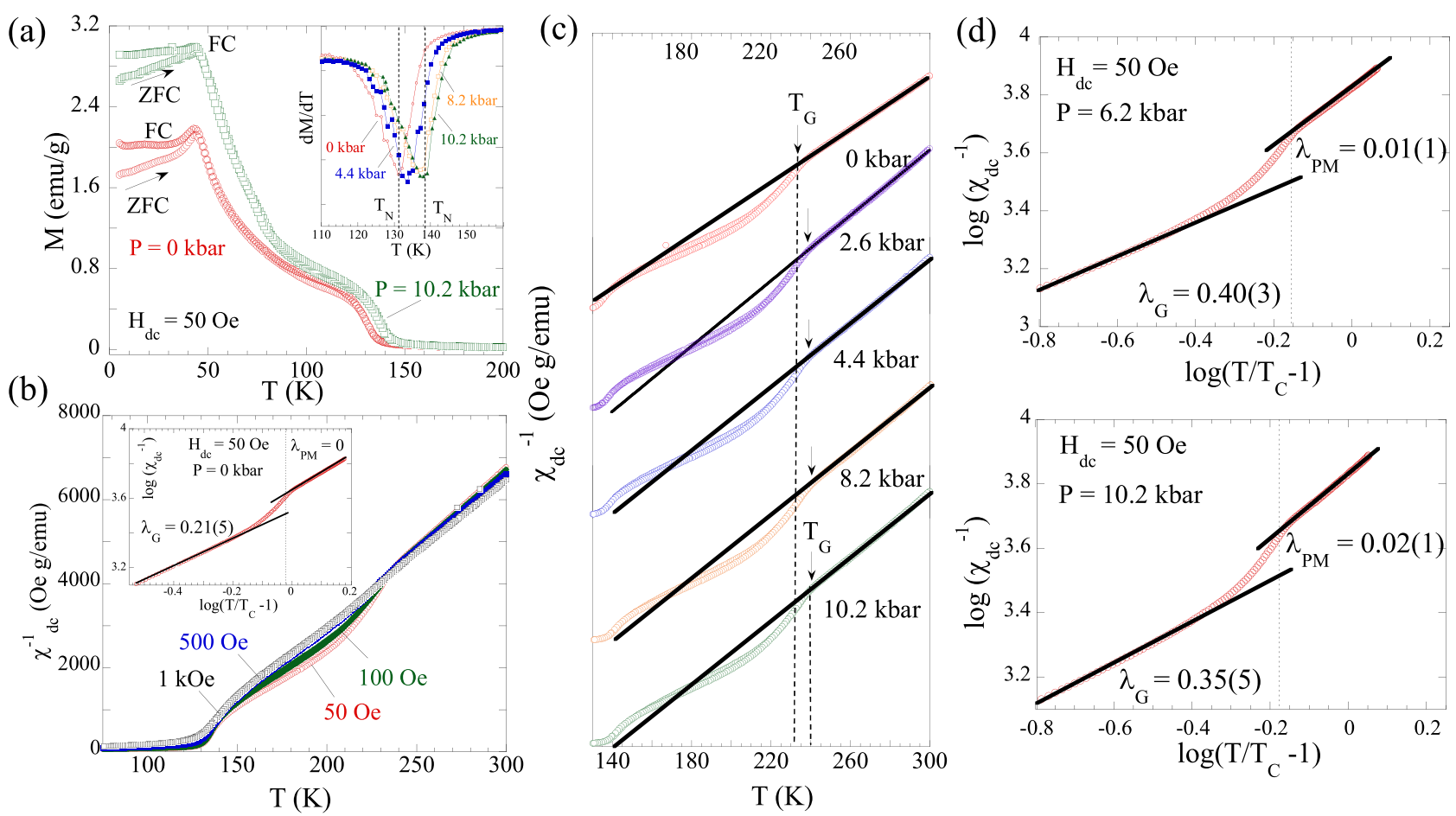

FIG. 3. Magnetization measurements as a function of temperature and hydrostatic pressure in $\mathrm{Gd}_{5} \mathrm{Ge}_{4}$. (a) ZFC and FC magnetizations as a function of temperature measured in an applied field of 50 Oe at $P=0$ kbar (circles) and under pressure of $10.2 \mathrm{kbar}$ (squares). Inset: $d M / d T$ at selected hydrostatic pressures. (b) $\chi_{d c}^{-1}(T)$ as a function of magnetic field, measured on heating. The inset shows fits at 50 Oe in the Griffiths and PM phases, respectively, at ambient pressures. (c) $\chi_{d c}^{-1}(T)$ at 50 Oe at selected hydrostatic pressures. The curves have been vertically displaced for clarity. Thick solid lines depict the Curie-Weiss fit to the inverse magnetic susceptibility at the PM state and the arrows show deviation from the $\mathrm{CW}$ law below the Griffiths temperature $T_{G}$ in each case. (d) Linear fit of $\chi_{d c}^{-1}$ vs $\left(T / T_{C}-1\right)$ in double logarithmic scale near $T_{G}$ at selected hydrostatic pressures (6.2 and 10.2 kbar).

and less distinguishable from the PM matrix becoming nearly indistinguishable from the high-temperature range values at $H_{d c}=1 \mathrm{kOe}$.

The effect of hydrostatic pressure on the onset of the GP in the PM regime is analyzed in Figs. 3(c) and 3(d). Figure 3(c) shows the $\chi_{d c}^{-1}(T)$ curves under selected pressures. The negative deviation from the Curie-Weiss law at the characteristic temperature $T_{G}$ is observed for all the measured pressures. Above $T_{G}$, all the susceptibility curves obey the Curie-Weiss law and yield a nearly equally effective magnetic moment $\left[\mu_{\text {eff }}=7.9(1) \mu_{B} / \mathrm{Gd}^{3+}\right]$. The value of $\theta_{P}$, however, increases as hydrostatic pressure increases (see Table II) reaching $\theta_{P}=$ $141(1) \mathrm{K}$ at the highest pressure (10.2 kbar). This is indicative of an enhancement of FM correlations. The origin of the positive deviations from linearity of the magnetic susceptibility for $P>2.6$ kbar observed in Fig. 3(c) can be ascribed to the coexistence of a small percentage of ferromagnetic ground state, induced by the hydrostatic pressure, with the majority antiferromagnetic one in $\mathrm{Gd}_{5} \mathrm{Ge}_{4}$ [23], although other possible origins cannot be discarded.

The fits of the logarithmic representation of $\chi_{d c}^{-1}(T)$ are shown in Fig. 3(d) for selected pressures $(P=6.2 \mathrm{kbar}$ and 10.2 kbar) as representative of this study on $\mathrm{Gd}_{5} \mathrm{Ge}_{4}$. It is worth noting that for each analysis the shift of $T_{C}$ with pressure has been taken into account. These measurements indicate that GP remains at higher pressures up to $10.2 \mathrm{kbar}$. The characteristic parameters obtained in this study are summarized in Table II. A slight variation of the exponent is observed in the investigated pressure range $\left[\lambda_{G}=0.21(5)\right.$ at $0 \mathrm{kbar}, \lambda_{G}=0.40(5)$ at $4.4 \mathrm{kbar}$, and $\lambda_{G} \sim 0.35(5)$ at $10.2 \mathrm{kbar}$, whereas $T_{G}$ shifts to higher temperatures with increasing pressure $\left[T_{G}=237(1) \mathrm{K}\right.$ at $0 \mathrm{kbar}$ and $T_{G}=$ 241(1) $\mathrm{K}$ at $10.2 \mathrm{kbar}]$.

Since the effect of both hydrostatic pressure and chemical pressure, i.e., Si doping (increasing $x$ ), is that of reducing the volume of the cell, again, we can compare the effects of hydrostatic pressure on GP with those reported by Ouyang et al. [13] in $\mathrm{Gd}_{5}\left(\mathrm{Si}_{x} \mathrm{Ge}_{1-x}\right)_{4}$ in the range of $0 \leqslant x \leqslant 0.875$. On the one

TABLE II. Magnetic properties of the $\mathrm{Gd}_{5} \mathrm{Ge}_{4}$ system obtained from the analysis of the low-field dc magnetic susceptibility at selected hydrostatic pressure values: the Néel temperature $T_{N}$, the Griffiths temperature $T_{G}$, the paramagnetic Curie temperature $\theta_{P}$, the susceptibility exponent $\lambda_{G}$, and the effective magnetic moment per $\mathrm{Gd}^{3+}$ ion $\mu_{\mathrm{eff}}^{P M} / \mathrm{Gd}^{3+}\left(\mu_{B}\right)$.

\begin{tabular}{lccccc}
\hline \hline $\begin{array}{l}P \\
\text { (kbar) }\end{array}$ & $\begin{array}{c}T_{N} \\
(\mathrm{~K})\end{array}$ & $\begin{array}{c}T_{G} \\
(\mathrm{~K})\end{array}$ & $\begin{array}{c}\theta_{P} \\
(\mathrm{~K})\end{array}$ & $\lambda_{G}$ & $\begin{array}{c}\mu_{\mathrm{eff}}^{P M} / \mathrm{Gd}^{3+} \\
\left(\mu_{B}\right)\end{array}$ \\
\hline 0 & 130 & 237 & 120 & $0.21(5)$ & $8.0(1)$ \\
2.6 & 133 & 238 & 137 & $0.32(3)$ & $7.9(1)$ \\
4.4 & 134 & 239 & 138 & $0.40(5)$ & $7.9(1)$ \\
6.2 & 135 & 240 & 139 & $0.40(3)$ & $7.9(1)$ \\
8.2 & 137 & 240 & 140 & $0.41(1)$ & $7.9(1)$ \\
10.2 & 138 & 241 & 141 & $0.35(5)$ & $7.9(1)$ \\
\hline \hline
\end{tabular}


hand, the hydrostatic pressure dependence of $T_{G}$ agrees with that reported by Ouyang et al. with chemical pressure $T_{G}(x)$ [13] (i.e., $T_{G}$ increases as cell volume decreases). According to that study, however, GP signatures were not detected in $\chi_{d c}^{-1}(T)$ above a critical concentration $x>0.5$. In that case, with increasing the Si content the negative deviation from Curie-Weiss at $T_{G}$ was found to decrease, becoming negligible for $x=0.875$. In the present study, however, the negative deviation observed in $\chi_{d c}^{-1}(T)$ below $T_{G}$ is not affected by the applied pressure [see Fig. 3(c)] and GP remains at higher pressures up to $10.2 \mathrm{kbar}$. On the other hand, according to Ouyang et al. [13] the parameter $\lambda_{G}$ was Si-content dependent (from $\lambda_{G}=0.62$ at $x=0$ to $\lambda_{G}=0$ at $x=0.5$ ). In the present study, however, a slight variation of the exponent $\lambda_{G}$ [from $\lambda_{G}=0.21(5)$ at $P=0 \mathrm{kbar}$ to $\lambda_{G}=0.35(5)$ at $P=10.5 \mathrm{kbar}]$ is observed in the investigated pressure range (see Table II). We can compare the cell volume reduction induced by hydrostatic pressure and doping $(x)$. From the value of the compressibility at low pressures taken from Magen et al. [23] $\kappa=-(1 / V) d V / d P=1.85(1) \mathrm{Mbar}^{-1}$, a hydrostatic pressure of $10 \mathrm{kbar}$ (the maximum pressure applied in our work) would reduce the unit cell volume of $\mathrm{Gd}_{5} \mathrm{Ge}_{4}$ to that of $x=0.5$ [31], and, therefore, according to the work by Ouyang [13], the presence of short range FM correlations in the PM state should not be observed. These results indicate that the effect of chemical pressure is significantly different to that of hydrostatic pressure, as the electronic changes associated with $\mathrm{Si} / \mathrm{Ge}$ doping are not taken into account in the pure volume reduction.

Figure 4 and Figure 5 display the dependence of the different phase transitions $T_{C, N}$ and $T_{G}$ with pressure obtained from the data shown in Fig. 2 and Fig. 3, respectively. Figure 4 displays the $T-P$ phase diagram for $\mathrm{Tb}_{4.925} \mathrm{La}_{0.075} \mathrm{Si}_{2} \mathrm{Ge}_{2}$. The M-PM $\rightarrow O(\mathrm{I})$-FM transition shifts linearly with pressure at a rate of $d T_{C} / d P=+2.0 \mathrm{~K} / \mathrm{kbar}$ on cooling and on warming. These values of the $d T_{C} / d P$ coefficient are much higher than those expected for second-order transitions [32]. Such a pressure-induced increase compares well with that reported at the first-order magnetostructural transition in $\mathrm{Gd}_{5} \mathrm{Si}_{1.8} \mathrm{Ge}_{2.2}\left(d T_{C} / d P=+3.0 \mathrm{~K} / \mathrm{kbar}\right.$ [32] $)$ and at the first-order structural transformation $T_{t}$ in $\mathrm{Tb}_{5} \mathrm{Si}_{2} \mathrm{Ge}_{2}$ $\left(d T_{t} / d P=+2.6 \mathrm{~K} / \mathrm{kbar}[19]\right)$. This fact together with the observed thermal hysteresis around $T_{C}$ points to the first-order nature of the transformation at $T_{C}$ in this compound in the pressure range studied. On the other hand, the application of pressure also moves $T_{G}$ linearly towards higher temperatures at a rate of $d T_{G} / d P=+1.0 \mathrm{~K} / \mathrm{kbar}$. Figure 5 shows the $T$ - $P$ phase diagram for $\mathrm{Gd}_{5} \mathrm{Ge}_{4}$. The $O$ (II)-PM $\rightarrow O$ (II)-AFM transition shifts linearly with pressure at a rate of $d T_{N} / d P=$ $+0.8 \mathrm{~K} / \mathrm{kbar}$, which corresponds to the expected rate for a second order transition, in agreement with previous works [23]. Regarding the $T_{G}$, a rate of $d T_{G} / d P=+0.4 \mathrm{~K} / \mathrm{kbar}$ is found in this case.

We can compare our results with those reported on the GP in $\mathrm{Dy}_{5} \mathrm{Si}_{3} \mathrm{Ge}$ [17], as a representative 5:4 compound of $\mathrm{M}-\mathrm{GP}$ with a second order M-PM $\rightarrow$ M-AFM transition. In that case, a moderate rate of $d T_{N} / d P=+0.34 \mathrm{~K} / \mathrm{kbar}$ was observed for the second order M-PM $\rightarrow$ M-AFM transition, whereas a slight increase of $T_{G}$ with pressure at a rate of $d T_{G} / d P=+0.16 \mathrm{~K} / \mathrm{kbar}$ was found.

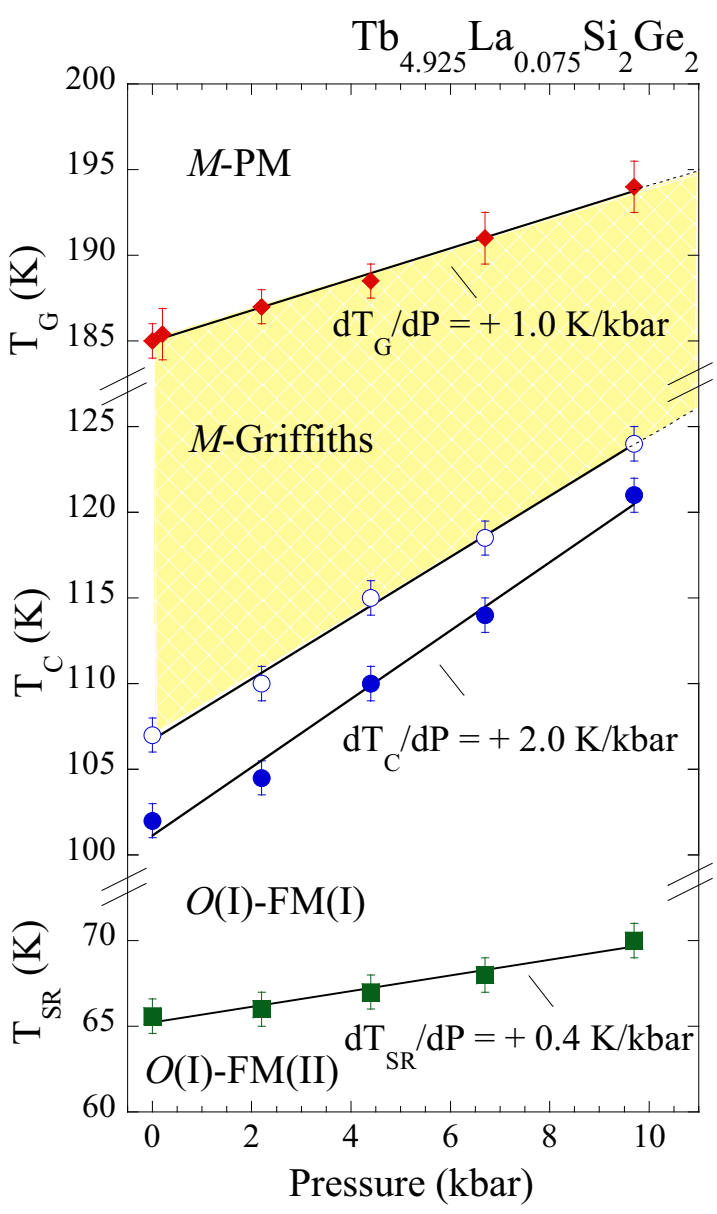

FIG. 4. T-P phase diagram as determined from magnetization measurements for $\mathrm{Tb}_{4.925} \mathrm{La}_{0.075} \mathrm{Si}_{2} \mathrm{Ge}_{2}$. Diamonds represent the onset of the GP phase $\left(T_{G}\right)$, circles represent the onset of the FM order $\left(T_{C}\right)$, and squares represent the spin reorientation transition $\left(T_{S R}\right)$. Open symbols are used in the warming curves $(\mathrm{FCW})$ and solid symbols in the cooling runs (FCC). Thick solid line depicts the magnetic and/or crystallographic phase boundaries. The curves have been vertically displaced for clarity.

In all the studied cases, the pressure-induced increase of the $T_{G}$ is lower than that of the corresponding long-range order transition $T_{C, N}$. The obtained rate values $d T_{G} / d P$ suggest a dependence on the nature of the long-range order (FM or AFM) transition: whereas a moderate pressure-induced increase of the $T_{G}$ is observed when $T_{C}, T_{N}$ are second order (e.g., $\mathrm{Gd}_{5} \mathrm{Ge}_{4}, \mathrm{Dy}_{5} \mathrm{Si}_{3} \mathrm{Ge}$ ), a much stronger effect is found when dealing with a first order nature of the transition at $T_{C}$ (e.g., $\mathrm{Tb}_{4.925} \mathrm{La}_{0.075} \mathrm{Si}_{2} \mathrm{Ge}_{2}$ ). In the latter, the simultaneous magnetic and structural change from M-PM to $O(\mathrm{I})-\mathrm{FM}$ at $T_{C}$ is accompanied by an enhancement of $d T_{C} / d P$ with respect to that expected for a second order pure magnetic transition [23]. The observed enhancement in $d T_{G} / d P$ in this compound can be due to a similar process occurring at $T_{G}$. According to that physical picture, the characteristic temperature where GP sets in $\left(T_{G}\right)$ in this compound would involve not only the formation of short-range ferromagnetic regions within the PM phase but also a structural change from $\mathrm{M}$ to $O(\mathrm{I})$. Thus the GP would consist of $O(\mathrm{I})$-FM clusters embedded in a 


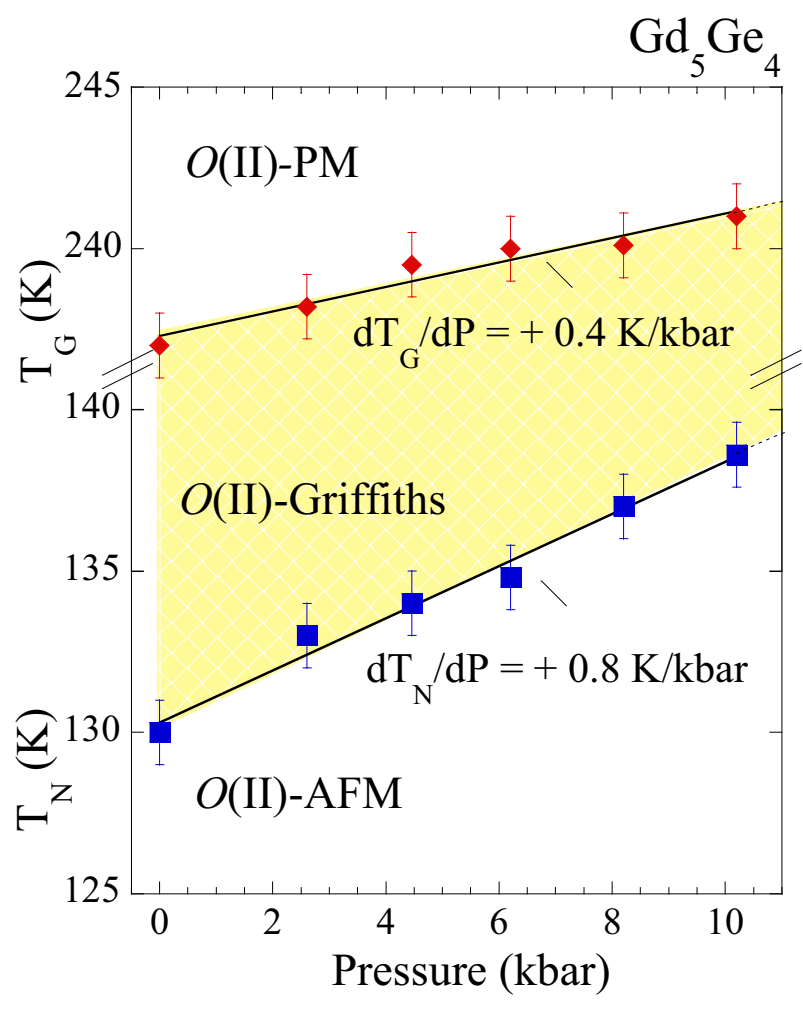

FIG. 5. T-P phase diagram, above $T_{N}$, as determined from magnetization measurements for $\mathrm{Gd}_{5} \mathrm{Ge}_{4}$. Diamonds represent the onset of the GP phase $\left(T_{G}\right)$ and squares are used for the AFM transition $\left(T_{N}\right)$. Thick solid line depicts the magnetic and/or crystallographic phase boundaries. The curves have been vertically displaced for clarity.

M-PM matrix, as it was previously proposed by Magen et al. [9]. This physical picture can also explain the lower rates $d T_{G} / d P$ observed in $\mathrm{Gd}_{5} \mathrm{Ge}_{4}$ and $\mathrm{Dy}_{5} \mathrm{Si}_{3} \mathrm{Ge}$ compounds. Unlike $\mathrm{Tb}_{4.925} \mathrm{La}_{0.075} \mathrm{Si}_{2} \mathrm{Ge}_{2}$, no structural changes are observed at $T_{C, N}$ for such low magnetic fields in $\mathrm{Gd}_{5} \mathrm{Ge}_{4}$ and $\mathrm{Dy}_{5} \mathrm{Si}_{3} \mathrm{Ge}$ compounds. Thus only a short-range magnetic order transition starts to develop at $T_{G}$ from $O$ (II)-PM to $O$ (II)-FM in $\mathrm{Gd}_{5} \mathrm{Ge}_{4}$ and from M-PM to M-AFM in $\mathrm{Dy}_{5} \mathrm{Si}_{3} \mathrm{Ge}$. According to the proposed physical picture, the clusters would maintain the $O(\mathrm{II})$ and $\mathrm{M}$ structural character of the paramagnetic state in these compounds.

In spite of such differences in the $d T_{G} / d P$ coefficients of the analyzed 5:4 compounds, a constant ratio $\frac{d T_{G} / d P}{d T_{C, N} / d P} \sim 0.5$ between the rates of the Griffiths-like temperature and the magnetic (FM or AFM) transition is found in all the studied compounds. Although the origin of such a behavior is not clear, we believe that it reflects the universality in the $T$ - $x$ phase diagrams of the $R_{5}\left(\mathrm{Si}_{x} \mathrm{Ge}_{1-x}\right)_{4}$ systems reported by Pereira et al. [12]. We note that the effect of pressure found on $T_{C}$ and $T_{G}$ on 5:4 intermetallics contrasts strongly with the decrease of both $T_{C}\left(d T_{C} / d P \sim-1 \mathrm{~K} / \mathrm{kbar}\right)$ and $T_{G}$ $\left(d T_{G} / d P \sim-3.6 \mathrm{~K} / \mathrm{kbar}\right)$ recently reported for disordered cobaltite $\mathrm{Gd}_{0.5} \mathrm{Sr}_{0.5} \mathrm{CoO}_{3-\delta}$ [33]. According to the authors, such a decrease can be ascribed to the well-known pressureinduced transition from the high-spin $\mathrm{Co}^{+3}$ state to the low-spin state in $\mathrm{Gd}_{0.5} \mathrm{Sr}_{0.5} \mathrm{CoO}_{3-\delta}$.

\section{CONCLUSIONS}

We have determined the T-P phase diagram of $\mathrm{Tb}_{4.925} \mathrm{La}_{0.075} \mathrm{Si}_{2} \mathrm{Ge}_{2}$ and $\mathrm{Gd}_{5} \mathrm{Ge}_{4}$ by means of low-field dc magnetization under hydrostatic pressure up to $10 \mathrm{kbar}$ in order to investigate the effect of pressure on the short-range magnetic correlations in the $\mathrm{M}$ and $O$ (II) structures for the 5:4 systems. The downward deviation in the inverse low-field dc susceptibility $\chi_{d c}^{-1}$ from the Curie-Weiss law below a characteristic temperature $T_{G}$ indicates that the Griffiths-like phase exists at pressures up to $10 \mathrm{kbar}$. The value of $T_{G}$, associated with the onset of short-range FM correlations, increases linearly with pressure. The differences observed in the $d T_{G} / d P$ coefficients of the analyzed 5:4 compounds suggest that the Griffiths temperature $T_{G}$ retains the nature (first or second order) of the main long-range magnetic order transition $T_{C, N}$. Further investigations are required, such as high-resolution x-ray diffraction and/or small angle neutron scattering on selected 5:4 compounds in order to unveil the structural signature of the GP phase. The universality found in the phase diagrams for this 5:4 family of materials by Pereira et al. [12] may be reflected in the ratio of $\sim 0.5$ between the pressure coefficients of the Griffiths-like and the Curie or Néel temperatures found in $\mathrm{M}$ and $O$ (II) structures. A comprehensive study on more compounds in this family is required in order to confirm this scenario. Theoretical models to explain the dependence of the Griffiths phases as a function of different external parameters, such as hydrostatic pressure, are lacking. We expect that this work may stimulate the interest of theoreticians in order to get a deeper insight on the interplay between external stimuli and the disorder-induced exotic magnetic behavior of Griffiths phases.

\section{ACKNOWLEDGMENTS}

Work at the University of Zaragoza is supported by the Spanish Ministerio de Ciencia, Innovación y Universidades through Project No. MAT2017-82970-C2-2-R and the Aragon Regional Government (Grant No. E28_20R). Work at the University of Cantabria is supported by the Spanish Ministerio de Ciencia, Innovación y Universidades through Project No. MAT2017-83631-C3-R. J.H.B. would like to thank FCT for Grant No. SFRH/BD/88440/2012, Project No. PTDC/FIS-MAC/31302/2017, and his Contract No. DL57/2016, Reference No. SFRH-BPD-87430/2012.
[1] R. B. Griffiths, Phys. Rev. Lett. 23, 17 (1969).

[2] A. J. Bray, Phys. Rev. Lett. 59, 586 (1987).

[3] J. Deisenhofer, D. Braak, H.-A. Krug von Nidda, J. Hemberger, R. M. Eremina, V. A. Ivanshin, A. M. Balbashov, G. Jug,
A. Loidl, T. Kimura, and Y. Tokura, Phys. Rev. Lett. 95, 257202 (2005).

[4] S. Zhou, Y. Guo, J. Zhao, L. He, and L. Shi, J. Phys. Chem. C 115, 1535 (2011). 
[5] M. B. Salamon, P. Lin, and S. H. Chun, Phys. Rev. Lett. 88, 197203 (2002).

[6] S. Guo, D. P. Young, R. T. Macaluso, D. A. Browne, N. L. Henderson, J. Y. Chan, L. L. Henry, and J. F. DiTusa, Phys. Rev. Lett. 100, 017209 (2008).

[7] M. Brando, D. Belitz, F. M. Grosche, and T. R. Kirkpatrick, Rev. Mod. Phys. 88, 025006 (2016).

[8] J. Herrero-Albillos, L. M. García, and F. Bartolomé, J. Phys.: Condens. Matter 21, 216004 (2009).

[9] C. Magén, P. A. Algarabel, L. Morellón, J. P. Araújo, C. Ritter, M. R. Ibarra, A. M. Pereira, and J. B. Sousa, Phys. Rev. Lett. 96, 167201 (2006).

[10] Z. W. Ouyang, V. K. Pecharsky, K. A. Gschneidner, Jr., D. L. Schlagel, and T. A. Lograsso, Phys. Rev. B 74, 094404 (2006).

[11] R. Nirmala, A. V. Morozkin, and S. K. Malik, Phys. Rev. B 75, 094419 (2007).

[12] A. M. Pereira, L. Morellón, C. Magén, J. Ventura, P. A. Algarabel, M. R. Ibarra, J. B. Sousa, and J. P. Araújo, Phys. Rev. B 82, 172406 (2010).

[13] Z. W. Ouyang, J. Appl. Phys. 108, 033907 (2010).

[14] W. Choe, V. K. Pecharsky, A. O. Pecharsky, K. A. Gschneidner, Jr., V. G. Young, Jr., and G. J. Miller, Phys. Rev. Lett. 84, 4617 (2000).

[15] C. Magén, L. Morellón, P. A. Algarabel, M. R. Ibarra, Z. Arnold, and C. Ritter, Advances in Solid State Physics (Springer, Berlin, 2007), Vol. 46, pp. 241-253.

[16] Y. Mudryk, V. K. Pecharsky, and K. A. Gschneidner, Jr., in Handbook on the Physics and Chemistry of Rare Earths, edited by J. C. G. Bünzli and V. K. Pecharsky (Elsevier, New York, 2014), Vol. 44, pp. 283-449.

[17] N. Marcano, P. A. Algarabel, J. R. Fernández, C. Magén, L. Morellón, N. K. Singh, K. A. Gschneidner, Jr., V. K. Pecharsky, and M. R. Ibarra, Phys. Rev. B 88, 214429 (2013).

[18] L. Morellón, C. Ritter, C. Magén, P. A. Algarabel, and M. R. Ibarra, Phys. Rev. B 68, 024417 (2003).

[19] L. Morellón, Z. Arnold, C. Magén, C. Ritter, O. Prokhnenko, Y. Skorokhod, P. A. Algarabel, M. R. Ibarra, and J. Kamarad, Phys. Rev. Lett. 93, 137201 (2004).
[20] J. H. Belo, A. M. Pereira, J. P. Araújo, C. de la Cruz, A. M. dos Santos, J. N. Gonçalves, V. S. Amaral, L. Morellón, M. R. Ibarra, P. A. Algarabel, and C. Magén, Phys. Rev. B 86, 014403 (2012).

[21] E. M. Levin, K. A. Gschneidner, Jr., and V. K. Pecharsky, Phys. Rev. B 65, 214427 (2002).

[22] C. Magén, L. Morellón, P. A. Algarabel, C. Marquina, and M. R. Ibarra, J. Phys.: Condens. Matter 15, 2389 (2003).

[23] C. Magén, Z. Arnold, L. Morellón, Y. Skorokhod, P. A. Algarabel, M. R. Ibarra, and J. Kamarad, Phys. Rev. Lett. 91, 207202 (2003).

[24] www.almax-easylab.com.

[25] V. O. Garlea, J. L. Zarestky, C. Y. Jones, L.-L. Lin, D. L. Schlagel, T. A. Lograsso, A. O. Tsokol, V. K. Pecharsky, K. A. Gschneidner, Jr., and C. Stassis, Phys. Rev. B 72, 104431 (2005).

[26] J. P. Araújo, A. M. Pereira, M. E. Braga, R. P. Pinto, J. M. Teixeira, F. C. Correia, J. B. Sousa, L. Morellón, P. A. Algarabel, C. Magén, and M. R. Ibarra, J. Phys.: Condens. Matter 17, 4941 (2005).

[27] N. Marcano, P. A. Algarabel, L. F. Barquín, J. P. Araujo, A. M. Pereira, J. H. Belo, C. Magén, L. Morellón, and M. R. Ibarra, Phys. Rev. B 99, 054419 (2019).

[28] A. H. Castro Neto, G. Castilla, and B. A. Jones, Phys. Rev. Lett. 81, 3531 (1998).

[29] Y.-C. Tseng, H.-J. Ma, C.-Y. Yang, Y. Mudryk, V. K. Pecharsky, K. A. Gschneidner, Jr., N. M. Souza-Neto, and D. Haskel, Phys. Rev. B 83, 104419 (2011).

[30] N. Pérez, F. Casanova, F. Bartolomé, L. M. García, A. Labarta, and X. Batlle, Phys. Rev. B 83, 184411 (2011).

[31] A. O. Pecharsky, K. A. Gschneidner, Jr., V. K. Pecharsky, and C. E. Schindler, J. Alloys Compd. 338, 126 (2002).

[32] L. Morellón, Z. Arnold, P. A. Algarabel, C. Magén, M. R. Ibarra, and Y. Skorokhod, J. Phys.: Condens. Matter 16, 1623 (2004).

[33] I. Fita, I. O. Troyanchuk, T. Zajarniuk, A. Wisniewski, and R. Puzniak, Phys. Rev. B 101, 224433 (2020). 